\title{
TERMINOLOGÍA DE URGENCIA Y GARANTÍAS PARA LA REPRESENTACIÓN TEMÁTICA: ELEMENTOS PARA EL ANÁLISIS DE DOMINIOS DE EMERGENCIA SÚBITA (DES).
}

\author{
TERMINOLOGY OF URGENCY AND GUARANTEES FOR \\ THE THEME REPRESENTACIÓN: ELEMENTS FOR THE \\ ANALYSIS OF SUDDEN EMERGENCY DOMAINS (DES)
}

Mario Baritéa

\begin{abstract}
RESUMO
Introdução: Os domínios emergentes são aqueles relativamente recentes, que se configuram como consequência da especialização, da progressiva interseção entre campos temáticos ou disciplinares pré-existentes ao surgimento de situações da realidade que requerem respostas inovadoras. O trabalho estuda a noção de domínio em organização do conhecimento e se propõe a um tipo particular de domínio emergente, o qual se denomina de domínio de emergência súbita (DES), entendido como qualquer nova área do conhecimento que nasce de imperativos da realidade, e de forma imprevisível. Objetivo: Contribuir para o embasamento teórico e caracterização dos DES, tomando para fins ilustrativos, o referencial do domínio gerado em torno do COVID-19. Metodologia: Uma análise em três níveis é proposta: sua denominação, sua terminologia aluvial e seu mapeamento. A análise é realizada a partir das perspectivas teóricas e metodológicas proporcionadas pela organização do conhecimento. Se destacam as abordagens centradas em análise de domínio, garantias e controle de vocabulário. Resultados: Os resultados, de natureza primária, têm como objetivo propor soluções específicas para representar tematicamente o DES. Conclusões: Dentre as conclusões, destaca-se que a organização do conhecimento possui um ponto de maturidade suficiente para enfrentar efetivamente os problemas terminológicos de sistematização e organização de conceitos apresentados por domínios emergentes e DES, em particular.
\end{abstract}

Descritores: Domínios de emergência súbita; Análise de domínio; Garantias; COVID19.

a Universidad de la República de Uruguay. E-mail: mario.barite@gmail.com

Inf. Inf., Londrina, v. 25, n. 3, p. 239 - 265, jul./set. 2020.

http://www.uel.br/revistas/informacao/ 


\section{INTRODUÇÃO}

Los dominios emergentes del conocimiento son aquellos relativamente recientes, que en general se configuran como consecuencia de la especialización (como sucede con la curaduría de arte), la progresiva intersección de campos temáticos o disciplinas preexistentes (como en el caso de los estudios métricos de la información, o de una de sus especies, la webometría), o por el surgimiento de situaciones de la realidad que requieren respuestas innovadoras desde las especialidades que pueden tomar a su cargo su análisis y desenvolvimiento (por ejemplo, la tecnología del streaming o lo relacionado con la detección de ondas gravitacionales).

En este trabajo se propone un tipo particular de dominio emergente, bajo la denominación de dominio de emergencia súbita (DES), entendido como toda área novísima del conocimiento que nace por imperativos de la realidad, y en muchos casos de forma imprevisible. Los DES suelen desairar todos los análisis predictivos y proyectivos, porque tienen su origen en fenómenos no esperados ni previstos. Es lo que ha sucedido, como ejemplo más inmediato, con la pandemia desatada por la aparición y rápida transmisión planetaria del virus SARS-CoV-2.

Los DES se constituyen, como todo dominio, a partir de un tópico central, en torno del cual se va instalando una constelación de términos que le orbitan. Algunos de ellos corresponden a conceptos nuevos, que surgen dentro del novel dominio o a partir del mismo, en tanto otros se extrapolan desde otros dominios, manteniendo, adaptando o modificando su concepto original. De una forma perentoria, entonces, surge y se expande la terminología de los DES, al tiempo que eclosiona la información y la documentación correspondiente, lo que impacta en la necesidad inmediata de contar con repertorios que recojan, organicen y relacionen esa terminología en el menor plazo posible (desde pequeños glosarios de divulgación hasta sistemas de organización del conocimiento que permitan indizar la numerosa documentación que se genera en plazos relativamente cortos).

Esa llamada 'terminología de urgencia' obliga a adaptar rápidamente 
métodos de la organización del conocimiento, para obtener obras de referencia y orientación. Presionan para ese fin tanto las exigencias y urgencias de los especialistas, como las de los mediadores de información (periodistas, divulgadores, diseñadores de campañas publicitarias, gestores de contenidos, bibliotecólogos, traductores).

En este trabajo se aspira a caracterizar genéricamente a los dominios de emergencia súbita (DES), a partir de una conceptualización -previa y actualizada- de los dominios del saber. A continuación se realizará un abordaje metodológico de caracterización de los DES en tres niveles:

i) el análisis del tópico que dará nombre al DES;

ii) la terminología del nuevo dominio, a partir de la identificación y recogida de términos propios del DES, y su necesaria justificación a través de alguna garantía.

iii) el mapeo del dominio, considerando las modalidades conocidas de análisis de dominio.

A efectos ilustrativos, se tomará como referencia el dominio relacionado con la enfermedad hoy llamada COVID-19, ocasionada por el ya mencionado virus SARS-CoV-2. Las conclusiones se presentarán como aportes a la mejor comprensión e interpretación de la naturaleza de los dominios en general y de los DES en particular, para favorecer la representación temática con fines de indización y recuperación de documentos e información.

\section{DOMINIOS}

En organización del conocimiento, la noción de dominio (domain en inglés) se corresponde con la idea de un "ámbito del saber o de la experiencia humana que se caracteriza por su especialización, o por la posibilidad de ser delimitado en función de las prácticas o los intereses propios de una comunidad de usuarios" (BARITÉ et al. 2015, p. 64).

Esta definición determina que todos los dominios son, en mayor o menor medida, especializados. Refieren a prácticas y conocimientos que comprenden a conjuntos limitados de personas, las que, por ese refinamiento en el manejo 
de un núcleo específico de conceptos, técnicas, aplicaciones y productos, pueden ser denominados, con justicia, especialistas.

La estructura interna de los dominios se va construyendo a lo largo del tiempo, a través de la interacción entre esas prácticas - que ya podemos denominar especializadas- y la consecuente reflexión sobre las mismas. Esta reflexión construida en los escenarios comunicacionales que generan y en las que participan los especialistas, provoca la formulación y puesta en cuestión de conceptos, premisas y principios generales, técnicas, métodos y formas reglamentarias (que abarcan desde una ley de ejercicio profesional hasta un código deontológico pasando por un reglamento interno, según las circunstancias).

Al poner en juego el conjunto de elementos arriba mencionados los especialistas terminan conformando una comunidad de discurso, un espacio regido por sus propias reglas y ambiciones, dentro del cual se configura un sublenguaje interno, o dicho de otra manera, una forma particular de expresar y desarrollar conceptos, una terminología propia (Swales 1987, 2016). Cada sublenguaje o lenguaje de especialidad constituye un subcódigo "parcialmente coincidente con el subcódigo de la lengua común, caracterizado en virtud de unas peculiaridades 'especiales', esto es, propias y específicas de cada uno de ellos, como pueden ser la temática, el tipo de interlocutores, la situación comunicativa, la intención del hablante, el medio en que se produce un intercambio comunicativo, el tipo de intercambio, etc." (CABRÉ, 1993, p. 128129).

No obstante, muchos dominios, en virtud de la naturaleza de sus objetos de estudio, y/o del impacto de sus prácticas y resultados en la vida comunitaria en sus dimensiones más relevantes (economía, cultura, sociedad, trabajo, salud), desbordan el ámbito de los especialistas y generan un desdoblamiento de sus universos: el interno y el que se configura extramuros, en diálogo -no siempre amable o pacífico- con la sociedad.

De este modo, hacia adentro del dominio continúan los procesos de evolución y acumulación del conocimiento con participación casi exclusiva de especialistas o de practicantes de dominios con los que guardan algún nivel de 
intersección.

La comunicación intramuros - extramuros, es decir, la que se establece entre el núcleo del dominio y el resto de la sociedad, suele darse a través de dos mecanismos tradicionales: la enseñanza, destinada a los aspirantes a participar regularmente en la vida interna del dominio, y la divulgación -sea científica o especializada- en sus distintas modalidades.

Modernamente, la divulgación se ha ido expresando en un discurso de ida y vuelta entre los especialistas y la sociedad, por "o crescente interesse público pela discussão e participação nas decisões sobre assuntos relacionados a C\&T", lo cual "parece apresentarse como imperativo de uma sociedade moderna - e que se quer democrática" (VALÉRIO; BAZZO, 2005, p. 2).

Cada dominio se organiza en torno a un tópico representativo de un problema o asunto que requiere ser explorado e investigado, para dar respuesta a asuntos que pueden no tener una conclusión definitiva o próxima. Un dominio puede ser tanto una macrodisciplina (las ciencias sociales), una disciplina (ciencias, tecnologías, disciplinas sociales y humanísticas, desde la biología a la teología), una subdisciplina (el derecho constitucional), una interdisciplina (las ciencias del medio ambiente), un área o un campo temático e incluso cualquier tópico de significación (la tecnología militar, los fenómenos migratorios, el tango o la viruela).

En las entrañas de los dominios macrodisciplinarios y disciplinarios hay espacio además para el surgimiento de múltiples dominios de menor dimensión y cuantía. Por ejemplo, cada una de las ramas de la medicina (la gastroenterología, la cirugía, la otorrinolaringología) se constituye en un dominio de relativa autonomía, pues si bien puede tomar para sí principios o conceptos generales de la disciplina común, cada rama está en condiciones de desarrollar su propio espacio, y en consecuencia, su propia terminología.

Aún más, un tópico aislado dentro del universo de la medicina (por ejemplo, la hidroterapia, las patologías tiroideas o incluso una cualquiera de las patologías tiroideas), puede formar un pequeño dominio, con sus propios fundamentos, métodos y objetivos.

Como señala Albrechtsen "the domain is not a kingdom, nor is it a republic. 
On the contrary, for the design of a KOS [knowledge organization system], the domain is, in the terminology of Schmidt and Wagner (2004) a 'field of work' for several interested parties (for instance, professional organizations, libraries or knowledge organization systems - KOS- researchers) that are concerned with the development of specific knowledge fields or tools" (ALBRECHTSEN, 2015, p. 559).

Un dominio es entonces, cualquier recorte de la realidad que pueda ser estudiado desde una o varias perspectivas, y en diferentes niveles de aproximación. Esta concepción de dominio es útil en organización del conocimiento porque cualquiera sea su dimensión (desde una macrodisciplina hasta un tópico de significación), será posible respaldar su existencia con especialistas y documentos específicos, y eventualmente con colecciones de datos y documentos especializados que requerirán ser organizadas para el acceso y la consulta.

A medida que se definen los dominios, van quedando primariamente determinados los productores y los usuarios principales, los documentos hábiles para servir de registro y base para la evolución del área, y las herramientas tecnológicas que permitirán la mediación (bancos de datos y bases de datos especializados). Por otra parte, se generan las condiciones para que puedan crearse bibliotecas especializadas en la temática, lo que constituye una de las formas calificadas de institucionalización de un dominio.

Configurado un dominio y creada su literatura de referencia, sus cultores principales y diversos actores externos requerirán sistemas nocionales que organicen los conceptos relacionados con el dominio (por ejemplo, clasificaciones científicas o taxonomías especializadas), así como sistemas de organización del conocimiento (tesauros, sistemas de clasificación, listas, taxonomías web) que cumplan con el propósito de etiquetar temáticamente y favorecer el acceso a los documentos, en cualquier formato en que se encuentren. Todo ello sin perjuicio de al menos otra docena de funciones que pueden cumplir en tanto "infraestructuras conceptuales" (SOERGEL, 2009). 


\section{DOMINIOS EMERGENTES Y DOMINIOS DE EMERGENCIA SÚBITA}

Los dominios emergentes, un tipo de dominio, son aquellos de desarrollo reciente (20 a 40 años), que son consecuencia del vertiginoso proceso de especialización y de intersección recíproca entre disciplinas y/o campos temáticos. En el caso de las especializaciones, en las primeras décadas de su existencia se encuentran en plena fase de conformación y especificación (ALTHUBAITY; AHMAD, 2002; HOEL; PAWLOWKI, 2012). Cuando se trata de intersección recíproca, como es el caso de la organización del conocimiento, en sus primeras etapas el dominio emergente puede estar reconstituyendo sus objetos de estudio y sus tendencias de pesquisa a partir de sus orígenes diversos, los que pueden ser relativamente antiguos (IYER, 2012; SMIRAGLIA, 2012).

La vertiginosa y continua aparición de dominios emergentes se inició en la inmediata posguerra, con la expansión de los estudios interdisciplinarios, el continuo desarrollo tecnológico (que ha mejorado de paso, las condiciones para la investigación científica en todas las áreas aplicadas, así como sus resultados), y la evolución del pensamiento intelectual orientado a cuestiones de sociedad y convivencia. Estos tres pilares han contribuido a la diversificación de los enfoques y la investigación sobre prácticamente cualquier asunto que tenga algún nivel de incerteza o controversia.

Los que aquí se propone denominar dominios de emergencia súbita (DES) se pueden considerar a priori como una variedad de dominio emergente, sobre todo por su irrupción imprevista, a partir de hechos de la realidad sobre los cuales no se había acumulado suficiente evidencia de predictibilidad. Suelen ser en su origen, consecuencia de intersecciones fulminantes entre distintas áreas del conocimiento a partir de hechos de la realidad, y solo en una fase posterior pueden desarrollarse como especializaciones.

Episodios tales como un fenómeno económico nuevo, el derribo de las torres gemelas, la aparición del sida o de distintas enfermedades virales de alto impacto sanitario son ejemplos de casos de los últimos 40 años, que generan un gran volumen de documentación en poco tiempo, así como un reacomodamiento 
de recursos humanos y materiales destinados a contener, interpretar y abordar desde distintas disciplinas los hechos y sus consecuencias, así como a establecer políticas preventivas y terapéuticas.

En la misma línea, una pandemia de las dimensiones de la COVID-19 es de las situaciones que llega a conmover en alguna medida los mapas del conocimiento. La vigilancia estadística permite el seguimiento cuantitativo y los cruces de datos. La valoración cualitativa ayuda a ponderar los daños y las proyecciones. Pero hay cuestiones más profundas en torno a esta pandemia, que alcanzan a la mayoría de las disciplinas de las ciencias naturales y humanas, la salud y la sociedad, imponiéndoles una renovada interpretación de la realidad. De este modo urgido e imprevisto, emerge un campo temático de estudio y de investigación (el que se constituye alrededor de la COVID-19), de rápida expansión, y con tantos vectores como disciplinas son afectadas.

Puede decirse que de alguna manera los DES se constituyen en dominios ad hoc, que pueden aprovecharse del conocimiento acumulado, al tiempo que generan en su torno un núcleo documentado de conocimientos específicos, los que con el paso de los años se estabilizan y sirven como referencia para fenómenos o situaciones similares.

La irrupción de los DES genera un problema de mayor cuantía para los gobiernos y también para distintos colectivos profesionales (periodistas, traductores, planificadores de salud). En el área de organización del conocimiento la mayor dificultad estriba en la avalancha de documentos, informaciones y datos que se acumulan rápidamente, y que necesitan ser procesados (esto es, descriptos, catalogados, clasificados e indizados) de inmediato, dado el repentino y urgente interés de distintas categorías de usuarios por el nuevo campo del saber.

La documentación que surge como consecuencia de la emergencia súbita de un nuevo ámbito de estudio tiene, además, un relativo nivel de fiabilidad, porque en las primeras fases de irrupción, prevalecen las interpretaciones, las hipótesis y las incertezas. Esa fiabilidad aumenta a medida que se agregan datos y nuevas evidencias. No obstante, ello obliga a una valoración constante de los documentos, en comunicación con especialistas y referentes que puedan 
orientar mejor la tarea profesional.

Si se propone la figura de los dominios de emergencia súbita como un concepto nuevo, es porque se considera que en términos de su naturaleza, su comprensión y de las estrategias para identificar su desarrollo y sus características se requieren respuestas un tanto diferentes a las habituales. Desde la organización del conocimiento, la identificación de un DES podría comprometer aspectos teóricos, técnicos y metodológicos para establecer las mejores estrategias de representación temática y de recuperación de datos, informaciones y documentos en los sistemas de información. Este punto es el que tratará de justificarse a través del presente trabajo.

\section{OBJETIVOS}

En función de lo expuesto en los apartados precedentes, en este trabajo se plantea como objetivo general, contribuir a la caracterización de los aquí llamados dominios de emergencia súbita (DES), tomando a efectos ilustrativos, la referencia del dominio generado en torno a la enfermedad conocida como COVID-19.

Se establecen asimismo los siguientes objetivos específicos:

Promover un fundamento teórico que dé primer sustento a la conceptualización de los dominios de emergencia súbita (DES).

Identificar en forma sumaria las dificultades que ofrecen los DES con relación a su denominación, al ingreso en aluvión de su terminología y a los mapeos que requieren para su mejor conocimiento e interpretación.

Identificar formas de análisis de dominio y garantías que contribuyan, desde la organización del conocimiento, a enfrentar las cuestiones de representación temática y la recuperación de información en los DES.

Vincular los elementos teóricos y metodológicos que se plantean, al DES que se ha ido conformando desde diciembre de 2019, en torno a la COVID-19. 


\section{METODOLOGIA}

El abordaje teórico y metodológico que se propone aquí para el análisis de un DES, parte del supuesto de que este tipo de dominio ofrece algunas particularidades tanto en lo relativo a su configuración, como en lo que hace a la instauración de sus términos y sus conceptos de referencia.

Esas particularidades obligan a tener en mente referencias generales a los aspectos teóricos y metodológicos vinculados a la organización de dominios, el control de vocabulario, la indización por lenguaje natural y cuestiones afines.

El análisis se dividirá en tres niveles relativos a la denominación, el mapeo y la terminología de un DES. Sobre esa estructura de base se realizará una primera aproximación al caso de la COVID-19, un DES surgido a fines del año 2019 que, ¿hace falta decirlo?, ha movido los cimientos de la vida social, económica, política y sanitaria del planeta.

\subsection{Denominación de Un DES}

El primer problema que plantea un dominio de emergencia súbita es el de su nombre, pues en un principio suelen prosperar diversas denominaciones, en diversos idiomas, producto de traducciones no siempre felices. Más allá de los matices y controversias que genera la afirmación de que el inglés es la lingua franca de la modernidad (JENKINS, 2009; SEIDLHOFER, 2009), lo cierto es que los primeros nombres que se implantan suelen presentarse en lengua inglesa. En las semanas y los meses subsiguientes comienzan a aparecer traducciones más o menos aceptadas en el resto de las lenguas, nuevas denominaciones que, para el conjunto de las lenguas latinas, siguen mayoritariamente las reglas de articulación de los formantes cultos, de cuño grecolatino (GUTIÉRREZ RODILLA, 1998, p. 118).

Las dudas y oscilaciones en la determinación de un nombre para un DES pueden afectar la comprensión inicial del nuevo fenómeno. Por otra parte, en torno al nombre de un dominio, considerado como término nuclear, se va organizando paulatinamente una constelación de términos relacionados, subordinados y cercanos al nuclear. Las ambigüedades del nombre del dominio 
afectan en mayor o menor medida la ubicación de cada término en el sistema nocional que está naciendo.

En caso de la COVID-19 puede afirmarse que para el común de la gente, el nombre del dominio corresponde tanto a la denominación actual del virus como de la enfermedad que provoca, lo que no se corresponde con la realidad.

Desde que el 20 de diciembre de 2019 fueran reportados los primeros casos de una neumonía de causa desconocida en la ciudad china de Wuhan, las denominaciones fueron sucediéndose con diferencia de pocos días, con relación al virus 2019-nCoV (acrónimo formado por el año, la primera letra de la palabra 'new' y una abreviatura de coronavirus), y a la enfermedad (infección por 2019nCoV, SARS de Wuhan -en referencia a un coronavirus anterior-, gripe de Wu y neumonía de Wuhan).

A la espera de un nombre oficial a ser asignado por los responsables de la Clasificación Internacional de Enfermedades, el 30 de enero de 2020, la Organización Mundial de la Salud (OMS) recomendó que el nombre provisorio de la dolencia fuera 'enfermedad respiratoria aguda por 2019-nCoV'.

Finalmente, el 11 de febrero de 2020 la OMS comunicó que el nombre definitivo de la enfermedad sería COVID-19, denominación que es una contracción de la expresión coronavirus disease 2019. En la fundamentación de la acuñación, se señaló la intención de que el nombre "did not refer to a geographical location, an animal, an individual or group of people, and which is also pronounceable and related to the disease" (ADHANOM, 2020).

El camino seguido por la OMS para la formalización del término, no impidió que de todas maneras aparecieran variantes menores de escritura como COVID 19 (sin guión), Covid-19, Covid 19 o sencillamente COVID en mayúscula y en minúscula. $O$ cuestiones de género: en algunos países hispanoamericanos se habla de la COVID, y en otros del COVID, presumiblemente por la confusión de la referencia al virus o a la enfermedad.

Curiosamente a nivel mundial prosperó el término 'coronavirus' como comprensivo del virus y de la enfermedad, lo que es técnicamente incorrecto. En efecto, coronavirus es un término genérico, acuñado en 1968. Debe su nombre a que bajo un microscopio electrónico, la superficie de un virus descubierto en 
esos años se parecía a la capa externa del Sol, llamada corona. Los coronavirus integran una familia de virus que pueden causar afecciones respiratorias graves tanto en animales como en humanos. De hecho, hacia mediados de los años sesenta del siglo pasado, una investigación desarrollada en el Reino Unido demostró que el primer coronavirus se correspondía con uno encontrado en pollos que padecían bronquitis, hacia el año 1930 (TYRRELL; BYNOE, 1965).

Desde el año 1968 hasta el presente se han catalogados siete tipos de coronavirus que afectan a los humanos, todos ellos nombrados por siglas 0 acrónimos. Los cuatro que en general provocan afecciones leves son 229E, OC43, NL63 y HKU1. Los otros tres pueden derivar en casos graves y en la muerte: MERS-CoV, SARS-CoV y SARS-CoV-2, este último responsable de la pandemia actual por COVID-19.

En aras de la precisión que puede alcanzarse a este momento, cabe decir entonces que COVID-19 es una enfermedad causada por un tipo de coronavirus cuya denominación oficial es SARS-CoV-2 (una fórmula difícil de recordar y de transcribir, salvo en entornos de alta especialización). A todos los efectos, parece razonable que el nombre más apropiado para el dominio sea COVID-19.

De todos modos, las imprecisiones que alcanzan a muchos de los potenciales usuarios de información en la denominación misma del dominio o en las distintas variantes de escritura que se dan, dificultan el acceso a documentos e información apropiada, pues las indizaciones y los etiquetados de contenidos tienen que obedecer del modo más ajustado a las nominaciones correctas de fenómenos, entidades y otros objetos de investigación.

En el caso concreto del dominio COVID-19 pueden ajustarse en los sistemas de información, los conceptos que se solapan y entremezclan en el conocimiento popular a través de operaciones habituales de control de vocabulario (control de sinonimia, homonimia y polisemia, y notas de alcance).

\subsubsection{Terminología de un DES}

Todo dominio, desde su origen, comienza un proceso paulatino de acumulación de conocimiento especializado que se expresa y se organiza a 
través de:

i) La construcción de los conceptos de referencia del dominio, de sus definiciones y alcances. Las formas de construcción de conceptos siguen reglas generales que son comunes a todos los dominios, conforme al modelo tradicional aristotélico, que permite la definición de los objetos de estudio, tan general o específicamente como sea necesario en cada contexto (veáse al respecto, BARITÉ 2017).

ii) La asignación de los términos que representarán a los conceptos. Para esta asignación se requiere apelar a alguna de las garantías aceptadas regularmente: literaria, de usuario, cultural, estructural, etc. (BULLARD, 2017).

iii) La identificación del sistema de relaciones que se establecen entre los conceptos, y por tanto, entre los términos que los representan.

iv) A partir de los conceptos, términos y relaciones del dominio, el establecimiento de un sistema nocional, que dé la base suficiente para la presentación de diversas estructuras terminológicas, entre ellas los sistemas de organización del conocimiento.

En suma, el sistema nocional de cualquier dominio se va construyendo por acumulación de conceptos que van siendo considerados, en forma más o menos pacífica, como propios de una comunidad de discurso, incluso en el caso de que tengan su origen en otros campos del saber. Esta comunidad de discurso los dota de sentidos particulares, y va estableciendo definiciones precisas y apropiadas a las necesidades de los especialistas. Los conceptos del dominio se relacionan en modalidades ya establecidas por la organización del conocimiento: a grandes rasgos, vínculos jerárquicos, vínculos no jerárquicos o asociativos, vínculos de sinonimia o cuasi sinonimia y vínculos funcionales.

Un problema central en este proceso es la elección de los términos que mejor pueden representar a los conceptos. En este punto, la noción de garantía adquiere trascendencia, pues conlleva el propósito de establecer -sobre el sistema nocional de base- una estructura terminológica donde cada término encuentra su lugar relativo, en función de los vínculos con otros. Las garantías elegidas auxilian en el proceso de elaboración colectiva de estructuras terminológicas consistentes, estables y representativas del sistema nocional 
construido por la comunidad de discurso.

Una definición clásica de garantía establece que "the warrant of a classification system can be thought of as the authority a classificacionist invokes first to justify and subsequently to verify decisions" acerca de la inclusión o exclusión de términos para representar conceptos en un dominio determinado (BEGHTOL, 1986, p. 110).

Bullard amplía el radio de aplicación de las garantías, desde el limitado universe de la creación de sistemas de clasificación, al señalar que "warrant is a common thread across a wide variety of systems ranging from traditional library classification to in -application menus and categories for web-based collections" [porque] "all designers of textual organizing schemas must look to some source for terminology" (BULLARD, 2017, p. 76).

Desde esta amplia perspectiva, la garantía debería ser vista como una herramienta útil para seleccionar, excluir o controlar terminología tanto para recursos convencionales como tesauros, como para la representación temática en sistemas de información o bases de datos, la elaboración de diccionarios y glosarios, o la organización terminológica de portales, repositorios y sitios web especializados.

Se han propuesto en la literatura de organización del conocimiento más de una veintena de garantías (BARITÉ, 2018, p. 528), desde que Hulme instaló el concepto de garantía literaria en 1911. Las más desarrolladas han sido la garantía cultural (LEE, 1976; BEGHTOL, 2002), la académica (BLISS, 1929; SACHS; SMIRAGLIA, 2004) y la de usuario (LANCASTER, 1977; HJØRLAND, 2013). Las garantías se distinguen por la apelación a distintas fuentes de autoridad para seleccionar y validar términos: la documentación en sentido amplio (garantía literaria), el lenguaje de comunidades con una identidad cultural o local que le es propia (garantía cultural), el vocabulario formal y representativo de las disciplinas (garantía académica), o las expresiones que utilizan los usuarios en sus búsquedas (garantía de usuario).

Las garantías constituyen filtros que regulan el ingreso a sistemas de información y otros contextos de información (páginas de prensa, documentos técnicos, traducción de documentos), del conjunto de términos que se refieren a 
un dominio concreto.

La utilización de garantías puede favorecer la consistencia interna de los mensajes en los distintos canales de comunicación por donde circula la información de los DES, que contiene en su discurso el aluvión de nueva terminología o terminología de urgencia. En efecto, las decisiones serán diferentes si hablamos de los circuitos de comunicación entre especialistas a veces con un discurso ultraespecializado, o de los que se utilizan para establecer una campaña pública de difusión y prevención.

La terminología de urgencia que acompaña al surgimiento de un DES suele reunir cuatro aristas que complican en particular los procesos de representación temática a través de terminología, para la clasificación, indización y organización de términos, o la creación de vocabularios controlados. Se trata de una terminología inestable, errática, ambigua y de valor temporal.

Inestable porque prosperan simultáneamente términos de distinta grafía y en distintos idiomas, para representar los conceptos que van surgiendo o se van rediscutiendo junto con el surgimiento del dominio. Errática porque no se establecen claramente acuerdos iniciales, ni siquiera entre especialistas, sobre los términos que se preferirán para la comunicación. Ambigua porque los mismos conceptos que son representados por los términos no están debidamente cristalizados y pueden ofrecer controversia. $Y$ de valor temporal porque un porcentaje significativo de los términos de urgencia suele tener corta vida, y son sustituidos por otros. La sucesión de nombres destinados a nominar el dominio mismo de la COVID-19, la confusión entre el nombre del virus y la enfermedad, y la resignada aceptación del nombre genérico del virus para representar a una variedad de ese género, como fue expuesto más arriba, es un ejemplo típico de estas calidades de la terminología de un DES.

La terminología de los DES se integra con: i) términos antiguos, que proceden del fondo común en el que abreva el nuevo espacio de conocimiento (por ejemplo, inmunidad residual, inmunidad de rebaño, que provienen de la epidemiología); ii) términos novedosos en forma y/o significado (por ejemplo, confinamiento social, PPE o personal protective equipment); iii) acepciones nuevas para términos ya existentes (por ejemplo, distanciamiento físico, 
distanciamiento social).

Al internacionalizarse, los términos comprendidos en el DES generan además problemas de traducción. Las distintas formas de nominar objetos y fenómenos por los especialistas, o por los divulgadores científicos, los periodistas, los bibliotecólogos, los traductores y otros mediadores, inciden también en la diversidad no siempre consistente de la terminología de los DES.

Por otra parte, desde hace al menos dos décadas, los usuarios de información prefieren acudir a Internet antes que a los sistemas de información, al menos para satisfacer en forma rápida sus más imperiosas necesidades de información. La consulta remota asistida por las distintas aplicaciones y funcionalidades que ofrecen los buscadores ha pasado a ser la forma habitual de búsqueda de información, lo que comprende también la búsqueda temática. En este contexto la apelación al lenguaje natural, con todas sus inconsistencias y su relativa confiabilidad, ha pasado a ser la opción más habitual.

La terminología que se asocia a la COVID-19 padece de esa heterogeneidad de situaciones, provocando ambigüedades, imprecisiones y dificultades en la comprensión y divulgación.

En el terreno estrictamente científico, los epidemiólogos, los virólogos, los químicos y los biólogos están jugando una carrera contra el tiempo, caracterizando al nuevo virus y su impacto en la salud, situando al agente y a la enfermedad en el mapa previo del conocimiento, y tratando de obtener los mejores resultados en cuanto a su identificación, forma de contagio, prevención y tratamiento.

Los profesionales de la salud, los responsables de la gestión hospitalaria y los prevencionistas llevan a terreno las conclusiones, siempre provisionales, que van llegando desde el mundo científico.

El diagnóstico y el tratamiento comprenden todavía un margen alto de incertidumbre, en virtud de la variabilidad de los síntomas (o la ausencia de ellos), la mutación del virus, y el desarrollo de formas terapéuticas que avanzan por acierto y error, y que parecen estar lejos de dar respuestas definitivas a la enfermedad.

Como la pandemia por COVID-19 ha tenido consecuencias en la 
economía, la cultura, la vida social, la educación y el mundo del trabajo, en cada uno de esos ámbitos suelen aparecer también algunos términos nuevos, que buscan etiquetar nuevas situaciones de la realidad (por ejemplo, infodemia).

Explorando solo a ras de agua, puede apreciarse que, a los efectos de establecer una política de control sobre el vocabulario propio de la COVID-19, proliferan los sinónimos o cuasi sinónimos. Por ejemplo, en los países latinoamericanos se utilizan al menos ocho denominaciones en lengua española para el mismo elemento de prevención: barbijo, máscara, mascarilla, tapaboca, tapabocas, cubreboca, cubrebocas y nasobuco.

A esta diversidad hay que sumar la que surge de las especificaciones: las mascarilla quirúrgica o la mascarilla con filtro puede variar su denominación conforme a su enlace sintáctico con los otros sinónimos: barbijo quirúrgico, cubrebocas quirúrgico, y así. Con este ejemplo, puede ilustrarse el amplísimo vocabulario alternativo que puede generarse a partir de un solo elemento de los tantos que integran el abanico de términos relativos solamente a la prevención.

\subsubsection{Mapeo de un DES}

Se entiende por mapeo el conjunto de procedimientos que se llevan a cabo para determinar el territorio semántico de un dominio, el conjunto de significados interrelacionados que refieren a su naturaleza, finalidad, objetos de estudio, herramientas y productos. Este conjunto de significados se procesa dentro de una comunidad de discurso, como ya fue dicho, asegurando la actualización, la vigencia y la comunicación de los conocimientos que le son propios.

El mapeo es, antes que nada, una técnica descriptiva que aporta una perspectiva macroestructural, de conjunto; permite establecer el estado de situación del dominio en un determinado momento. Como técnica puede utilizarse para propósitos diversos, y de sus resultados se pueden inferir enfoques interpretativos y proyectivos.

Parece obvio señalar que el dominio preexiste al mapeo, y por ello el mapeo obtiene una descripción organizada, como resultado de una 
interpretación del sistema nocional que se ha instaurado en el dominio. Sin embargo, en los dominios de emergencia súbita, esa puede ser una verdad relativa, porque la emergencia de los conceptos y de los vínculos que se van estableciendo entre ellos, puede darse en forma concomitante con el desarrollo vertiginoso del sistema nocional que le dará sustento epistemológico y semántico.

Las operaciones de mapeo en organización del conocimiento suelen realizarse a través de alguno de los abordajes de análisis de dominio (domain analysis) que se dirán, $Y$ en su expresión más simplificada ofrecen una distribución gráfica de términos y relaciones, soportada por los conceptos propios del dominio: su sistema nocional. En palabras de Albretchsen, "as a 'field of work' the domain is constructed in and through the process of planning, design and construction of a particular KOS" (Albrechtsen, 2015, p. 559).

La conceptualización de lo que entendemos por análisis de dominio se ha ido desarrollando a lo largo de veinticinco años, particularmente en torno a las contribuciones de Albrechtsen y, sobre todo, del investigador dinamarqués Birger Hjørland (ALBRECHTSEN, 1993; HJØRLAND; ALBRECHTSEN, 1995; HJØRLAND, 2002; 2017). Fue él quien identificó y propuso once modalidades de análisis de dominio: producción de guías de literatura; construcción de clasificaciones especializadas y tesauros; indización y recuperación de información en áreas especializadas; estudios de usuarios; estudios bibliométricos; estudios históricos; estudios documentales y de tipos documentales; estudios epistemológicos y críticos; estudios terminológicos; estructuras e instituciones en la comunicación científica; y, cognición científica, conocimiento experto e inteligencia artificial (HJØRLAND, 2002).

Posteriormente Smiraglia sugirió la posibilidad de retirar de esa lista a la indización y recuperación de información en áreas especializadas, y a las estructuras e instituciones en la comunicación científica, al tiempo que sugirió la inclusión de la semántica de las bases de datos y el análisis del discurso (SMIRAGLIA, 2015, p. 97). En el mismo año se propuso incorporar a las once modalidades iniciales la procedencia u origen (provenance), como una forma de análisis de dominio típica de la Archivología (GUIMARÃES; TOGNOLI, 2015). 
Cabe preguntarse qué formas de análisis de dominio pueden realizar mejor el mapeo de un DES, considerando su forma de emergencia. En efecto, un DES irrumpe en el mundo del conocimiento cual una isla volcánica que emerge repentinamente en el océano, como resultado de alguna erupción submarina. Va definiendo su territorio, cambiante cada día, por sucesivos aluviones, y por la paulatina y nerviosa estabilización de sus materiales. Con el paso de los años, irá constituyéndose en un dominio emergente primero, y en un dominio común del conocimiento después, pero en su primera fase de desarrollo, los mapeos del DES que puedan realizarse tendrán la misma inestabilidad y dificultad que su estructura nocional y su terminología.

López-Huertas (2015) ha analizado las particularidades de la aplicación de las modalidades de análisis de dominio a los dominios interdisciplinarios, situación que puede ser parcialmente asimilable al análisis de los DES, puesto que muchos de los dominios de emergencia súbita tienen componentes multidisciplinarios e interdisciplinarios desde su origen. Esta autora señala que los métodos más efectivos para el mapeo de dominios interdisciplinarios son "indexing and retrieving specialties, terminological studies, constructing special classifications and thesauri, bibliometrical studies, empirical user studies, document and genre studies and epistemological and critical studies" (LÓPEZHUERTAS, 2015, p. 575), y argumenta con detalle acerca de cada modalidad.

Se puede aventurar que la semántica de las bases de datos puede ser también una alternativa fiable para los mapeos de DES. En las bases de datos y en otras fuentes digitales es, probablemente, donde queda mejor registrada y accesible la terminología iniciática y cambiante de los DES. Al mismo tiempo, las funcionalidades de los motores de búsqueda y las aplicaciones suelen permitir estudios cuantitativos y cualitativos que constituyen una buena base para la organización paulatina del dominio.

De todos modos, no cabría desdeñar los estudios que pueden realizarse desde la lingüística de corpus aplicada a la terminología (BEVILACQUA, 2013), para compilar el conjunto de voces nuevas que integra un DES al mundo del conocimiento. El obstáculo mayor que quizás deba superarse desde esta perspectiva, es que las fuentes informatizadas de donde puede extraerse 
información de un DES suelen ser heterogéneas, y con distintos niveles de fiabilidad, incluso en fuentes de procedencia científica o técnica.

En efecto, la rápida y sucesiva modificación de las hipótesis y las explicaciones relativas a los fenómenos poco entendidos de un DES, generan incertidumbre respecto a la calidad y la confiabilidad de las terminologías y los conceptos que ellas representan.

Como puede deducirse de lo expuesto, los sistemas de organización del conocimiento (tesauros, sistemas de clasificación, listas de descriptores o de encabezamientos de materias) enfrentan serias dificultades para ir incorporando los términos novedosos que surgen en torno a un DES. La dificultad más evidente estriba en que, entre la publicación de una edición y la siguiente de un sistema de organización del conocimiento, pueden pasar diez años. Por ese motivo, las posibilidades que se tienen para responder en tiempos razonables a las demandas inmediatas de documentos e información fiable sobre un DES, pasan por combinar una serie de estrategias locales, como por ejemplo:

i) el seguimiento, la recolección y la comprensión de los términos que proporciona el lenguaje natural;

ii) la conformación de listas alfabéticas de palabras clave, y de anillos de sinónimos, que sirvan como primeros términos de indización, los cuales serán seguramente- susceptibles de cambios.

iii) el establecimiento de vínculos jerárquicos y asociativos entre términos, cuya identificación vaya enriqueciendo el control de vocabulario de esas listas.

Los enfoques deductivos o bottom-up parecen ser los más apropiados para ir recogiendo y cribando la terminología de un DES, con vistas a su organización sistemática, para fines de recuperación de información.

Para los clasificacionistas y los indizadores, la organización paulatina de la terminología de un DES debe ser paciente y metódica. Para favorecer la eficiencia y la eficacia de los procesos, conviene comprometerse en proyectos cooperativos que lleven adelante organizaciones internacionales, regionales o nacionales, o redes y sistemas de bibliotecas, para contribuir a la utilización de una terminología suficientemente aceptada y fundamentada.

Las garantías que parecen más adecuadas en estas situaciones son la 
garantía literaria y la garantía académica.

La garantía literaria se apoya en la terminología que ofrece la documentación entendida en sentido amplio (publicaciones convencionales o digitales, portales, repositorios, sitios web, medios de prensa).

La garantía académica combina y aprovecha la organización científica o especializada previa, el consenso o el acuerdo de los especialistas respecto al alcance de cada concepto, y la opinión experta. Las clasificaciones científicas previas son importantes a este respecto, porque permiten situar a un DES en el entorno temático que le corresponde, y favorecen la identificación de los puentes que pueda tener con otras disciplinas o especialidades

El caso del DES relativo a COVID-19 es expresivo de las dificultades mencionadas. A un año de la aparición del virus, los tipos documentales que proveen cierta expresión de conjunto del dominio COVID-19 en este primer año de pandemia, son los glosarios y los materiales de divulgación, destinados a mediadores en la comunicación (ORGANIZACIÓN PANAMERICANA DE LA SALUD, 2020; UNIVERSITY OF VIRGINIA, 2020) o a público en general (Zhou 2020). No existen en cambio, clasificaciones o diccionarios que puedan dar cuenta de la avalancha de términos que se han ido acumulando en tan breve plazo.

En cualquier caso, un posible camino hacia el mapeo del DES COVID-19, podría apoyarse en el siguiente esquema de partida:

i) términos propios de la biología, la virología y la epidemiología, aplicables a las cuestiones de la COVID-19; este grupo seguramente reúna el mayor número de expresiones;

ii) términos relativos a la medicina sus cuatros grandes vertientes: anatomía, fisiología, patología, tratamiento;

iii) términos tomados a préstamo de las ciencias humanas y sociales; algunos de ellos seguramente han sido reformulados para atender las especificidades de los fenómenos sociales asociados al dominio COVID-19. 


\section{CONCLUSOES}

En este trabajo se enfatiza en la noción de dominio como expresiva del conjunto de las disciplinas científicas (ciencias puras y aplicadas, ciencias de la naturaleza, ciencias sociales y humanidades), las tecnologías, los oficios, los deportes y cualquier otro ámbito que se organiza en torno a prácticas especializadas y a asuntos que generan documentación en una medida significativa, a partir de la actividad regular y en evolución, de una comunidad de práctica y de discurso.

Esta perspectiva amplia es importante para la organización del conocimiento, porque permite develar y sistematizar el conjunto de las características que son comunes a todos los dominios, más allá de su mayor o menor dimensión, antigüedad, cientificidad, tecnología, especificidad, intersección y comunicación con otros dominios.

Los dominios emergentes, y en especial los dominios de emergencia súbita presentan, sin embargo, algunas particularidades que afectan las posibilidades de acceso y recuperación a información y documentación específica a los mismos.

Los DES ofrecen el desafío de desencadenar, en breves períodos, una andanada (o catarata, tsunami, riada, todas las analogías son válidas) de conceptos que, o son totalmente novedosos, o provienen de distintas matrices disciplinarias, y en su conjunto tratan de dar cuenta de las numerosas perspectivas de análisis y sistematización conceptual que requieren estos dominios de irrupción imprevista.

Como quedó expuesto, un abordaje posible desde las teorías y los métodos establecidos en la organización del conocimiento, puede darse en tres niveles de análisis: la identificación del término tope que identificará al DES, la situación de su terminología en el estudio particularizado término a término, y el mapeo del nuevo dominio, con la pretensión de construir sistemas de clasificación, tesauros u otras formas de sistemas de organización del conocimiento, que permitan organizar la caótica y vertiginosa acumulación de información y documentación, bajo criterios de pertinencia, confiabilidad y 
estabilidad conceptual.

Puede afirmarse que la organización del conocimiento se encuentra en un punto de madurez suficiente desde los puntos de vista teórico y metodológico, como para enfrentar con eficacia los problemas terminológicos, de sistematización y de organización de conceptos que plantean los dominios emergentes en general, y los dominios de emergencia súbita en particular.

Herramientas tales como las distintas formas de análisis de dominio y de control de vocabulario, y las garantías de legitimación y validación de términos, aplicadas consistentemente tanto respecto a expresiones del lenguaje natural como a la formación de glosarios y vocabularios controlados de urgencia, pueden contribuir a organizar la conformación errática de los primeros sistemas nocionales en este tipo de dominios.

La utilización del dominio COVID-19 como caso ilustrativo de los problemas identificados para la organización conceptual y terminológica de los DES permite confirmar la necesidad de acudir a herramientas tradicionales de la organización del conocimiento, tanto para apoyar el trabajo de los científicos como para colaborar en la divulgación de los avances registrados en el conocimiento del nuevo dominio.

\section{REFERÊNCIAS}

ADHANOM, Tedros. WHO Director-General's remarks at the media briefing on 2019-nCoV on 11 February 2020. Disponível em:

https://www.who.int/dg/speeches/detail/who-director-general-s-remarks-at-themedia-briefing-on-2019-ncov-on-11-february-2020

ALBRECHTSEN, Hanne. This is not domain analysis. Knowledge Organization, 42(8), 557-561. 2015.

ALBRECHTSEN, Hanne. Subject analysis and indexing: from automated indexing to domain analysis. The Indexer, 18(4): 221-224. 1993.

AL-THUBAITY, AbdulMohsen; KHURSHID, Ahmad. Tracking knowledge of emergent domains. In: Proceddings: Sixth IEEE International Conference on Information Visualisation, London, 10-12 July 2002. Guildford: University of Surrey. 
BARITÉ, Mario. Literary warrant. Knowledge Organization, 45(6): 517-536. 2018.

BARITÉ, Mario. La definición en Terminología. In: Álvarez Catalá, Sara y Mario Barité (Orgs.). Teoría y praxis en Terminología. Montevideo: CSIC. 2017.

BARITÉ, Mario et al. Diccionario de Organización del Conocimiento:

Clasificación, Indización, Terminología. 6a edición ampliada y revisada. Montevideo: CSIC. 2015.

BEGHTOL, Clare. Universal concepts, cultural warrant and cultural hospitality. Advances in Knowledge Organization, 8, p: 45-49. 2002.

BEGHTOL, Clare. Semantic validity: concepts of warrant in bilbliographic classification systems. Library Resources \& Technical Services, 30(2): 109123. 1986.

BEVILACQUA, Cleci. Por que e para que a linguística de corpus na terminologia. In Tagnin, Stella e Cleci Bevilacqua (Orgs.). Corpora na terminologia. São Paulo: Hub, 11-27. 2013.

\section{BLISS, Henry. E. The Organization of Knowledge and the System of the} Sciences. New York: Holt. 1929.

BULLARD, Julia. Warrant as a Means to Study Classification System Design. Journal of Documentation, 73(1): 75-90. 2017.

CABRÉ, María Teresa. La terminología: teoría, metodología, aplicaciones. Barcelona: Antártida/Empúries. 1993.

GUTIÉRREZ RODILLA, Bertha M. La ciencia empieza en la palabra: Análisis e historia del lenguaje científico. Barcelona: Península. 1998.

GUIMARÃES, José Augusto Chaves; TOGNOLI, Natália Bolfarini. Provenance as a domain analysis approach in archival knowledge organization. Knowledge Organization, 42(8): 562-569. 2015.

HOEL, Tore; JAN M., Pawlowki. Managing standards development in emergent fields of technology innovation - a proposed model of key processes in ICT standardization. In: Enterprise Interoperability: Proceedings of the Workshops of the Sixth International Conference. I-ESA '12, ISTE Ltd, London, 2012.

HJØRLAND, Birger. Domain Analysis. Knowledge Organization, 44(6): 436464. 2017.

HJØRLAND, Birger. Theories of knowledge organization-theories of knowledge. Knowledge Organization, 40(3): 169-181. 2013. 
HJØRLAND, Birger. Domain analysis in information science: eleven approaches - traditional as well as innovative. Journal of documentation 58: 422-462. 2002.

HJØRLAND, Birger; ALBRECHTSEN, Hanne. Toward a new horizon in information science: domain-analysis. Journal of the American Society for Information Science, 46(6), 400-425. 1995.

IYER, Hemalata. Classificatory structures: concepts, relations and representation. Würzburg: Ergon Verlag. 2012.

JENKINS, J. English as a lingua franca: interpretations and attitudes. World Englishes, 28(2): 200-207. 2009.

LANCASTER, F. W. Vocabulary control in information retrieval systems. Advances in Librarianship, 7: 1-40. 1977.

LEE, J. M. E. Wyndham Hulme: a reconsideration. In: Rayward, W.B. (Ed.). The Variety of Librarianship: Essays in Honour of John Wallace Metcalfe. Sydney: LAA. 1976.

LÓPEZ-HUERTAS, María J. Domain analysis for interdisciplinary knowledge domains. Knowledge Organization, 42(8), 570-580. 2015.

ORGANIZACIÓN PANAMERICANA DE LA SALUD. COVID-19: glosario sobre brotes y epidemias: un recurso para periodistas y comunicadores. 2020. Disponível em: https://www.paho.org/es/node/70518

SACHS, Moshe; SMIRAGLIA, Richard P. From encyclopedism to domainbased ontology of knowledge management: the evolution of the Sachs Classification (SC). Advances in Knowledge Organization, 9: 167-172. 2004.

SCHMIDT, Kjeld; INA, Wagner. Ordering systems: coordinative practices and artifacts in architectural design and planning. Computer Supported Cooperative Work (CSCW), 13: 349-408. 2004.

SEIDLHOFER, B. Common ground and different realities: world Englishes and English as a lingua franca». World Englishes, 28(2): 236-245. 2009.

SMIRAGLIA, Richard P. Domain analysis for knowledge organization: tools for ontology extraction. Oxford: Chandos Publishing. 2015.

SMIRAGLIA, Richard P. Knowledge organization: some trends in an emergent domain. El profesional de la información, 21(3): 225-227. 2012.

SOERGEL, Dagobert. Knowledge organization systems: overview. 2009. Disponível em: http://www.dsoergel.com/SoergelKOSOverview.pdf 
SWALES, John M. Reflections on the concept of discourse community. ASp, 69: 7-1. 2016.

SWALES, John M. Approaching the concept of discourse community. In: 38th Annual Meeting of the Conference on College Composition and Communication, Atlanta, GA, March 19-21 1987. S.I.: ERIC. 1987.

TYRRELL, D. A. J.; Bynoe, M. L. Cultivation of a novel type of common cold virus in organ culture, British Medical Journal, 1:1467-1470. 1965.

UNIVERSITY OF VIRGINIA. Coronavirus \& COVID-19: Glossary of Terms. UVA Health, Charlottesville, VA: UVA. 2020. Disponível em: https://uvahealth.com/services/covid19-glossary

VALÉRIO, Marcelo; BAZZO,Walter Antonio. O papel da divulgação científica em nossa sociedade de risco: em prol de uma nova ordem de relações entre ciência, tecnologia e sociedade. In: XXXIII Congresso Brasileiro de Ensino da Engenheria: COBENGE, 2005. Campina Grande, PB. Disponível em: http://www.abenge.org.br/cobenge/arquivos/14/artigos/SC-10-299879209001117474585219.pdf

ZHOU, Wang. Manual de prevención del coronavirus: 92 consejos basados en la evidencia científica que pueden salvar tu vida. S.I.: Alienta, 2020.

\title{
TERMINOLOGY OF URGENCY AND GUARANTEES FOR THE THEME REPRESENTACIÓN: ELEMENTS FOR THE ANALYSIS OF SUDDEN EMERGENCY DOMAINS (DES).
}

\begin{abstract}
Introduction: The emerging domains are those that are relatively recent, which are configured as a consequence of specialization, of the progressive intersection between pre-existing thematic or disciplinary fields to the emergence of reality situations that require innovative responses. The work studies the notion of domain in knowledge organization and proposes a particular type of emerging domain, which is called the sudden emergency domain (DES), understood as any new area of knowledge that is born from imperatives of reality, and unpredictably. Objective: To contribute to the theoretical basis and characterization of DES, taking for illustrative purposes, the referential of the domain generated around COVID-19. Methodology: A three-level analysis is proposed: its name, its alluvial terminology and its mapping. The analysis is carried out from the theoretical and methodological perspectives provided by the organization of knowledge. The approaches centered on domain analysis, guarantees and vocabulary control stand out. Results: The results, of primary nature, aim to propose specific solutions to thematically represent DES. Conclusions: Among the conclusions, it is highlighted that the knowledge organization has a sufficient maturity point to effectively face the terminological problems of systematization and organization of concepts presented by emerging domains and DES, in particular.
\end{abstract}


Descriptors: Sudden emergency domains; Domain analysis; Warranties; COVID-19.

\begin{abstract}
RESUMEN
Introducción: Los dominios emergentes son aquellos que son relativamente recientes, los cuales se configuran como consecuencia de la especialización, de la progresiva intersección entre campos temáticos o disciplinarios preexistentes a la emergencia de situaciones de realidad que requieren respuestas innovadoras. El trabajo estudia la noción de dominio en la organización del conocimiento y propone un tipo particular de dominio emergente, que se denomina dominio de emergencia repentina (DES), entendido como cualquier nueva área de conocimiento que nazca de imperativos de la realidad, y impredeciblemente. Objetivo: Contribuir a la base teórica y caracterización de los DES, tomando a título ilustrativo, el referencial del dominio generado en torno a COVID-19. Metodología: Se propone un análisis de tres niveles: su nombre, su terminología aluvial y su cartografía. El análisis se realiza desde las perspectivas teóricas y metodológicas que brinda la organización del conocimiento. Destacan los enfoques centrados en el análisis de dominios, garantías y control de vocabulario. Resultados: Los resultados, de carácter primario, tienen como objetivo proponer soluciones específicas para representar temáticamente el DES. Conclusiones: Entre las conclusiones, se destaca que la organización del conocimiento tiene un punto de madurez suficiente para afrontar eficazmente los problemas terminológicos de sistematización y organización de conceptos presentados por dominios emergentes y DES, en particular.
\end{abstract}

Descriptores: Dominios de emergencia repentina; Análisis de dominio; Garantías; COVID-19.

Recebido em: $15 / 09 / 2020$

Aceito em: 30/09/2020 